EPJ Web of Conferences 97,00008 (2015)

DOI: 10.1051/epjconf/ 20159700008

(C) Owned by the authors, published by EDP Sciences, 2015

\title{
$S=-1$ meson-baryon interaction in hot and dense nuclear mat- ter: chiral symmetry, many-body and unitarization for a road to GSI/FAIR
}

\author{
Daniel Cabrera ${ }^{1,2, a}$, Laura Tolos ${ }^{2,3, b}$, Jörg Aichelin ${ }^{4, c}$, and Elena Bratkovskaya ${ }^{1,2, d}$ \\ ${ }^{1}$ Institut für Theoretische Physik, Johann Wolfgang Goethe-Universität, Max-von-Laue-Straße 1, 60438 \\ Frankfurt am Main, Germany \\ ${ }^{2}$ Frankfurt Institute for Advanced Studies, Ruth-Moufang-Straße 1, 60438 Frankfurt am Main, Germany \\ ${ }^{3}$ Institut de Ciències de l'Espai (IEEC/CSIC), Campus Universitat Autònoma de Barcelona, Facultat de Cièn- \\ cies, Torre C5, E-08193 Bellaterra, Spain \\ ${ }^{4}$ Subatech, UMR 6457, IN2P3/CNRS, Université de Nantes, École des Mines de Nantes, 4 rue Alfred \\ Kastler, 44307 Nantes cedex 3, France
}

\begin{abstract}
The dynamics of strange pseudoscalar and vector mesons in hot and dense nuclear matter is studied within a chiral unitary framework in coupled channels. Our results set up the starting point for implementations in microscopic transport approaches of heavy-ion collisions, particularly at the conditions of the forthcoming experiments at GSI/FAIR and NICA-Dubna. In the $\bar{K} N$ sector we focus on the calculation of (off-shell) transition rates for the most relevant binary reactions involved in strangeness production close to threshold energies, with special attention to the excitation of sub-threshold hyperon resonances and isospin effects (e.g. $\bar{K} p$ vs $\bar{K} n$ ). We also give an overview of recent theoretical developments regarding the dynamics of strange vector mesons $\left(K^{*}, \bar{K}^{*}\right.$ and $\phi$ ) in the nuclear medium, in connection with experimental activity from heavy-ion collisions and nuclear production reactions. We emphasize the role of hadronic decay modes and the excitation of hyperon resonances as the driving mechanisms modifying the properties of vector mesons.
\end{abstract}

\section{Introduction}

The features of strongly interacting matter in a broad range of temperatures and densities has been a subject of great interest in the last decades, in connection with fundamental aspects of the strong interaction such as the nature of (de)confinement or the physical mechanism of chiral symmetry restoration. Hadrons with strangeness, in particular, have been matter of intense investigation regarding the study of exotic atoms [1], the analysis of strangeness production in heavy-ion collisions (HICs) [2-4] and the microscopic dynamics ruling the composition of neutron stars [5].

\footnotetext{
a e-mail: cabrera@ fias.uni-frankfurt.de

be-mail: tolos@ice.csic.es

ce-mail: Aichelin@subatech.in2p3.fr

de-mail: Elena.Bratkovskaya@th.physik.uni-frankfurt.de
}

This is an Open Access article distributed under the terms of the Creative Commons Attribution License 4.0, which permits unrestricted use, distribution, and reproduction in any medium, provided the original work is properly cited. 
Understanding the dynamics of light strange mesons in a nuclear environment has posed a challenge to theoretical models, amongst different reasons, due to the failure of the low-density approximation to describe the interaction of the $\bar{K}$ meson with the medium. The $\Lambda(1405)$ resonance, excited right below the $\bar{K} N$ threshold in vacuum, is shifted to upper energies in nuclear matter due to Pauliblocking on the nucleons. This implies a rapid change of the $\bar{K} N$ scattering amplitude from repulsive in vacuum to attractive in the medium already at low densities, as it is required by the phenomenology of kaonic atoms [1].

The properties of both $K$ and $\bar{K}$ close to threshold energy have also been thoroughly investigated in HICs at SIS energies [2-4, 6]. The analysis of experimental data in conjunction with microscopic transport approaches has allowed to draw solid conclusions regarding the production mechanisms and the freeze-out conditions of strange mesons. A good agreement with data has been achieved for many different observables when the in-medium properties of kaons are implemented. Still, a simultaneous description of all observables involving antikaon production is missing [4]. For example, recent experimental data on the $v_{1}, v_{2}$ flow coefficients of strange mesons show sensitivity to the details of the in-medium meson-baryon interaction, leaving room for a more elaborated description of the most relevant reactions (e.g., $\pi Y \rightarrow \bar{K} N$ ) within hadronic models.

Strange vector meson resonances $\left(\bar{K}^{*}, K^{*}\right)$ have triggered substantial attention recently, both theoretically and experimentally. An early motivation for present theoretical developments originates in the substantial experimental activity within the RHIC low energy scan programme and the HADES experiment at GSI, which are currently performing measurements in order to extract the in-medium properties of hadronic resonances and, in particular, of strange vector mesons from HICs [7, 8]. Unlike their unflavoured partners, strange vector mesons do not decay in the dilepton channel, making their experimental detection less clean. Still, there is hope that their in-medium properties can be accessed via reconstruction of their hadronic decays. Presumably, the lack of baryonic resonances with $S=+1$ calls for much more moderate effects on the $K^{*}$ and thus a scenario of non-degenerate spectral functions for the $K^{*}$ and $\bar{K}^{*}$ emerges in a baryonic medium. Presently, there is also a big interest in the experimental study of $K^{*}, \bar{K}^{*}$ production at ultrarelativistic energies since the vector strange resonances are considered as a promising probe of the hot medium and the quark-gluon plasma (QGP) formation [9].

With the focus on the implementation of effective hadronic models based on chiral dynamics in microscopic transport simulations, we report in this talk our recent results on $\bar{K} N$ scattering in hot and dense matter as well as on the spectral properties of strange vector mesons at the experimental conditions to be met in low and intermediate energy HICs (e.g. GSI/FAIR, NICA-Dubna).

\section{$2 \bar{K} N$ interaction in hot and dense nuclear matter}

The properties of strange mesons in nuclear matter at finite temperature were studied within a self-consistent coupled-channel approach based on the $S U(3)$ meson-baryon chiral Lagrangian (see $[10,11]$ and references therein). Such kind of approaches successfully describe the $\bar{K}$ meson interactions in nuclear matter, accounting for a shallow attractive $\bar{K}$ potential at nuclear matter density (40-60 MeV) and a broad spectral function. Our present results improve on this model by implementing the unitarization of scattering amplitudes in both the $s$ and $p$ waves of the $\bar{K} N$ interaction, at finite density and temperature, within the imaginary time (Matsubara) formalism [12]. The scattering amplitudes are modified in the medium from Pauli-blocking on intermediate nucleon states, Bose enhancement of light mesons, mean-field baryonic binding potentials and the self-energy of mesons (particularly, pions and kaons in, e.g. $\bar{K} N, \pi Y$ ). The latter leads to a selfconsistent solution of the coupled-channel Bethe-Salpeter equations, $T=V+V G T$, determining the meson-baryon scattering 
amplitude or $T$-matrix. Here $V$ contains the meson-baryon potential from the leading-order chiral Lagrangian and $G$ stands for the meson-baryon resolvent or loop function. $G$ depends on the $\bar{K}$ selfenergy through the meson propagator, which at the same time is evaluated by summing the effective $\bar{K} N$ interaction over the nucleon Fermi distribution, schematically $\Pi_{\bar{K}}=\sum_{\vec{p}} n(\vec{p}) T_{\bar{K} N}$. The $T$-matrix (also referred to as $\mathcal{G}$-matrix when the medium is switched on) fulfills unitarization along the righthand cut, extending the applicability of the chiral effective theory to higher energies and, particularly, to the presence of resonant states. Such is the case of the $\Lambda(1405)$, which is dynamically generated in the $s$-wave.

The present extension not only generalizes the results of [10] to hot and dense matter, but additionally gives access to full off-shell in-medium scattering amplitudes in the $S U(3)$ set of coupled channels, both in $s$ and $p$ waves. For $K^{-} p$ scattering these include (in the basis of physical states): $K^{-} p, \bar{K}^{0} n, \pi^{0} \Lambda, \pi^{0} \Sigma^{0}, \eta \Lambda, \eta \Sigma^{0}, \pi^{+} \Sigma^{-}, \pi^{-} \Sigma^{+}, K^{+} \Xi^{-}$and $K^{0} \Xi^{0}$; whereas for $K^{-} n$ scattering we have $K^{-} n, \pi^{0} \Sigma^{-}, \pi^{-} \Sigma^{0}, \pi^{-} \Lambda, \eta \Sigma^{-}$and $K^{0} \Xi^{-}$. We note that the $\eta$ and $\Xi$ channels where not included in the analysis of [6], based on an implementation of $\bar{K} N$ dynamics from the Jülich meson-exchange model in the Giessen-Frankfurt Hadron-String-Dynamics (HSD) transport model. Another important asset of our approach is that, whereas it achieves a good description of $K^{-} p$ vacuum low-energy scattering observables (such as cross sections, scattering lengths and branching ratios at threshold) with a minimal number of parameters, the model is highly predictive also in the $K^{-} n$ sector. It has been pointed out [4] that isospin effects could be essential to understand strangeness production close to threshold energies. Therefore, having a hold on the reactions off the proton and the neutron is a strong point of the model. To summarize, our calculation delivers unitarized amplitudes for all the coupled channels in the $K^{-} p$ and $K^{-} n$ sectors, in partial waves $L=0,1$ and total angular momentum $J=1 / 2,3 / 2$. According to their isospin, the following resonances are excited: in the $s$-wave, we have the $\Lambda(1405)$ with $I=0$ and $J=1 / 2$; in $p$-wave we account for the $\Lambda(1115)$ with $I=0$ and the $\Sigma(1195)$ with $I=1$, both with total $J=1 / 2$, and the $\Sigma^{*}(1385)$ with $I=1$ and $J=3 / 2$.

The calculation of dynamical quantities in transport theory requires that the pertinent reaction rates or transition probabilities are folded with the spectral functions of the particles in the initial and final states [6]. We discuss below these transition probabilities including medium effects in hot and dense matter for several $\bar{K} N$ two-body processes which play a key role in accessing the near sub-threshold region in antikaon production dynamics.

The transition probability for a given reaction, $\mathcal{P}(s)$, is defined as the angular integrated modulussquared scattering amplitude (including all partial waves, $L=0,1$ ), averaged over the total angular momentum $(J=1 / 2,3 / 2)$. In our approach it can be defined fully off-shell as a function of the total energy $P^{0}$ and momentum $\vec{P}$ of the meson-baryon pair. For the process $i \rightarrow j$ (where $i, j$ denote meson-baryon channels, as listed above) one has

$$
\mathcal{P}_{i j}\left(P^{0}, \vec{P} ; \rho, T\right)=\int_{-1}^{+1} d(\cos \theta)\left\{\left|f_{i j}^{(s)}+\left(2 f_{i j}^{+}+f_{i j}^{-}\right) \cos \theta\right|^{2}+\left|f_{i j}^{+}-f_{i j}^{-}\right|^{2} \sin ^{2} \theta\right\},
$$

where $f^{(s)}$ stands for the $s$-wave amplitude $\left(f^{(s)}=T^{L=0}\right), f^{ \pm}$correspond to the $p$-wave amplitudes for total angular momentum $J=L \pm 1 / 2$, and $\theta$ is the scattering angle in the center of mass (c. m.) frame of the meson-baryon pair. For transport models which rely on the narrow test quasiparticle approach, such as IQMD, it is pertinent to make the connection between these transition probabilities and the cross section by means of an on-shell reduction scheme [i.e. fixing a dispersion relation for $\left.\left(P^{0}, \vec{P}\right)\right][4,6]$. For completeness, the cross section for the process $i \rightarrow j$ reads $\sigma_{i j}=\frac{1}{8 \pi} \frac{M_{i} M_{j}}{s} \frac{\tilde{q}_{j}}{\tilde{q}_{i}} \mathcal{P}_{i j}$, with $\tilde{q}_{i}$ the c.m. three-momentum and $M_{i}$ the baryon mass in channel $i$. The total cross section for a given reaction (e.g. $K^{-} p$ ) follows as the sum over the partial cross sections for all the permitted coupled channels, $\sigma_{\text {tot }}=\sum_{j} \sigma_{i j}$. We emphasize here that in our model we also have access to the 

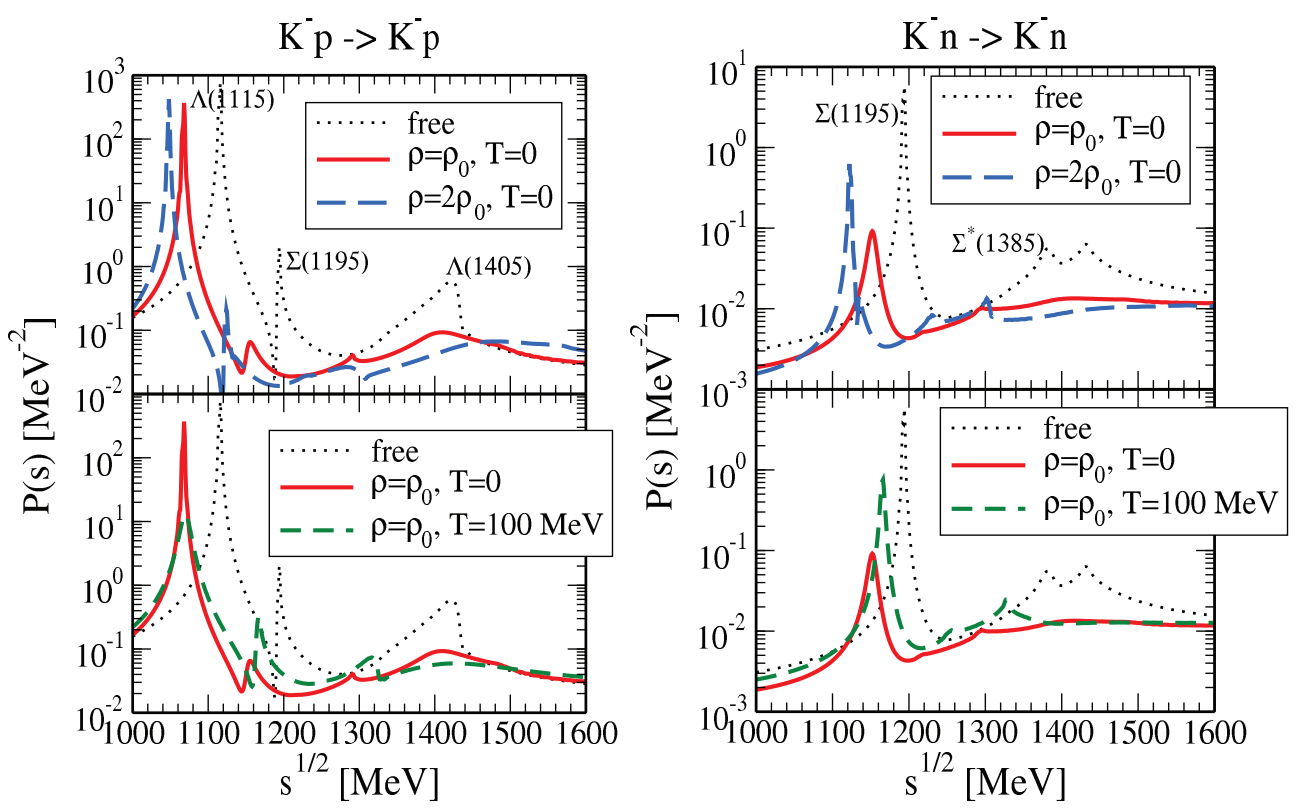

Figure 1. In-medium transition probability $\mathcal{P}$ at zero total three-momentum for the elastic $K^{-} p$ (left) and $K^{-} n$ (right) reactions. The peaks associated to the $\Lambda(1115), \Sigma(1195)$ and $\Lambda(1405)$ resonances are clearly visible in the vacuum case.

angular dependence of the scattering amplitudes. Although the total cross sections are dominated by the $s$-wave interaction, due to the contribution of the (deeply) subthreshold hyperon excitations in the $p$-wave the angular differential cross section is non-trivial and $\sigma_{\text {tot }}$ exhibits anisotropy. The angular information is a novel aspect of our implementation of strangeness dynamics in transport models and allows for a full determination of the kinematics of the outgoing particles.

In Fig. 1 the transition probability for the $K^{-} p$ and $K^{-} n$ elastic reactions is depicted as a function of the meson-baryon energy at total vanishing three-momentum, evidencing the importance of isospin effects. The $K^{-} p$ state is an admixture of isospin $I=0,1$ and therefore the two isoscalar $\Lambda$ resonances and the isovector $\Sigma(1195)$ show up in the spectrum. The $\Sigma^{*}(1385)$ couples weakly to the $\bar{K} N$ system and cannot be resolved in the $K^{-} p$ elastic reaction. The $K^{-} n$ reaction is pure $I=1$ and consequently only the isovector hyperon excitations are present in the right panel of Fig. 1. This explains the dramatic difference between the $K^{-} p$ and $K^{-} n$ total cross sections in vacuum, the former being dominated by a quick fall due to a strong Flatté effect on the resonance shape of the $\Lambda(1405)$, who sits immediately below the $K^{-} p$ threshold. The hyperon profiles exhibit the temperature and density evolution as dictated by the scattering amplitudes in our model, which can be summarized as follows: the structure of the $\Lambda(1405)$ is practically washed out and only some remnants are visible already at normal matter density. The $p$-wave ground states experience moderately attractive mass shifts as density is increased, whereas the $\Sigma^{*}(1385)$ is largely broadened due to the opening of in-medium decay channels (e.g. $\Sigma^{*} \rightarrow \Lambda N N^{-1}, \Sigma N N^{-1}$ ) related with the selfenergy of pions and kaons (the $\Sigma^{*}$ evolution is better appreciated in the right panel of Fig. 2). The effect of temperature is particularly appreciable as a broadening of the $p$-wave resonances as compared to the vacuum case. A pole analysis of 

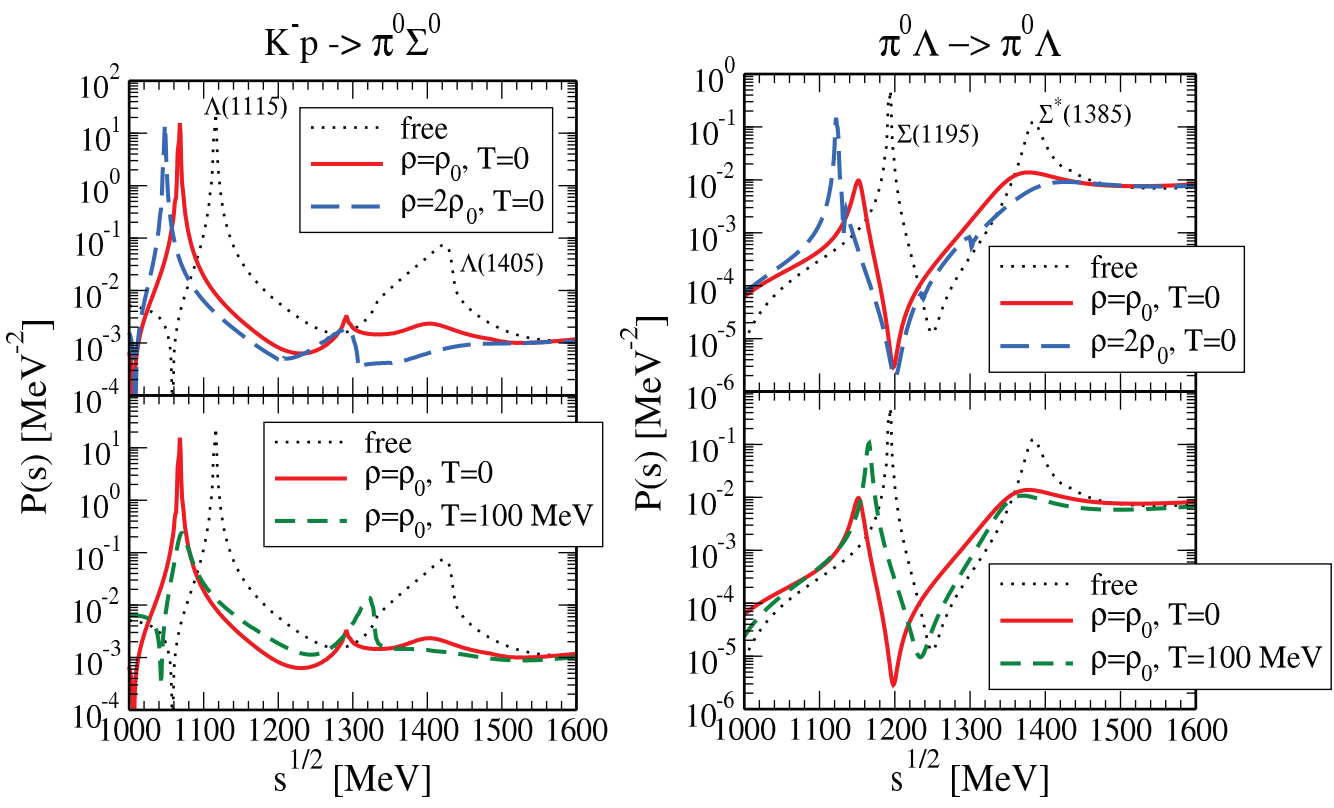

Figure 2. Same as in Fig. 1 for the inelastic $K^{-} p \rightarrow \pi^{0} \Sigma^{0}$ and $\pi^{0} \Lambda \rightarrow \pi^{0} \Lambda$ reactions.

the scattering amplitudes permits to obtain momentum-dependent nuclear optical potentials for these hyperons at different temperatures and densities (cf. [12] for full details).

The inelastic reactions $K^{-} p \rightarrow \pi^{0} \Sigma^{0}$ and $\pi^{0} \Lambda \rightarrow \pi^{0} \Lambda$ are accounted for in Fig. 2. The $\pi^{0} \Sigma^{0}$ channel selects the $I=0$ component of the $\bar{K} N$ amplitude and therefore only the isoscalar hyperons show up in the reaction rate. Conversely, the $\pi^{0} \Lambda$ reaction is a pure isovector process and only the $\Sigma$ resonances populate the spectrum. Here one appreciates the large in-medium width of the $\Sigma^{*}$ as induced by the one- and two-body mechanisms incorporated through the dressing of pions and kaons, and the small change in the position of the resonance, in agreement with previous determinations in cold nuclear matter [10, 13].

Our results are complementary to the ones obtained in our previous work [11], where the $\bar{K}$ spectral function and nuclear optical potential were provided. Altogether, they permit a systematic accounting of medium effects in the $S=-1$ sector within transport approaches such as PHSD and IQMD, not only regarding binary reactions but also the production and propagation of light strange hadrons.

\section{Strange vector mesons: $K^{*}, \bar{K}^{*}$ and $\phi$}

On the theory side, the properties of $\bar{K}^{*}$ have been studied in cold nuclear matter [17] starting from a model of the $\bar{K}^{*} N$ interaction within a unitary chiral Lagrangian approach in coupled channels [14-16], extended to account for the coupling to vector mesons from the $J^{P}=1 / 2^{-}$octet in the Hidden Local Symmetry formalism. The $\bar{K}^{*}$ selfenergy receives two contributions associated to (i) its dominant decay mode $\bar{K}^{*} \rightarrow \bar{K} \pi$ and (ii) the quasi-elastic reaction $\bar{K}^{*} N \rightarrow \bar{K}^{*} N$ and related absorption channels. The latter mechanism induces a strong broadening of the $\bar{K}^{*}$ spectral function as a result of the mixing with two $J^{P}=1 / 2^{-}$states, the $\Lambda(1783)$ and $\Sigma(1830)$, which are dynamically generated in 

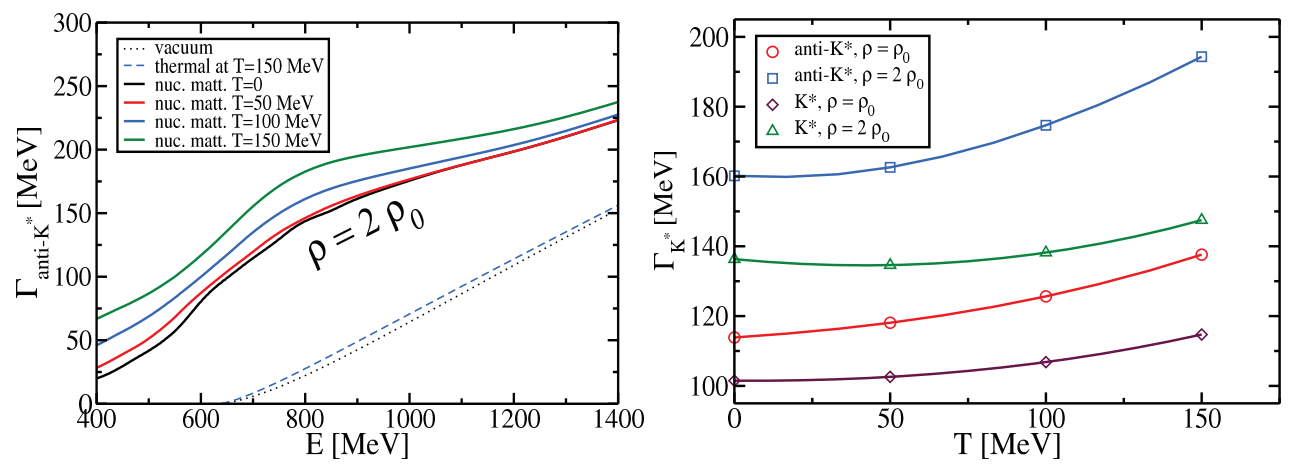

Figure 3. Left: Imaginary part of the $\bar{K}^{*}$ selfenergy from $\bar{K} \pi$ decays as a function of the energy at nuclear density $\rho=2 \rho_{0}$ and several temperatures. Right: Temperature evolution of the $\bar{K}^{*}\left(K^{*}\right)$ width due to $\bar{K}(K) \pi$ in-medium decays at $\rho=\rho_{0}, 2 \rho_{0}$.

a parallel way to the $\Lambda(1405)$ in $\bar{K} N$ scattering. At $\rho=\rho_{0}$, the $\bar{K}^{*}$ width is enlarged beyond $200 \mathrm{MeV}$, the in-medium $\bar{K} \pi$ cloud contributing about $50 \%$.

The situation for the $K^{*}$ meson is similar to that of the $K$ and one expects milder changes in the presence of a nuclear medium. The lack of resonant states close to threshold advises to use a t $\rho$ approximation for the $K^{*}$ selfenergy. The calculation within the chiral approach of [17] is straightforward at threshold energy and leads to a positive $K^{*}$ mass shift of about $\Delta m_{K^{*}}\left(\rho_{0}\right) \simeq 30 \mathrm{MeV}[18,19]$. Additional progress on the theoretical description of the $K^{*} N$ interaction has been reported recently in [20], where the dynamics of the coupled system $K^{*} N-K N$ system is explored from a extension of the Kroll-Ruderman interaction by replacing the photon by a strange vector meson. The coupled dynamics of $K$ and $K^{*}$ improves notably the description of the isoscalar and isovector $K N$ phase shifts up to energies of about $2 \mathrm{GeV}$. The latter opens the possibility for an immediate extension of our previous calculation of the $K^{*}$ selfenergy $[18,19]$ beyond threshold energies, thus giving access to a realistic evaluation of the $K^{*}$ momentum-dependent optical potential.

Interest in the $\phi$ meson properties has also been revived in recent years. The $\phi$, having a hidden strangeness content, strongly couples to the $\bar{K} K$ system and therefore its in-medium dynamics is highly governed by its decay into the light pseudoscalars (its coupling to the nucleon is OZIforbidden). The production of $\phi$ 's in HICs is being investigated both at RHIC and at the LHC, with special attention to the ratio between dilepton and hadronic decays [21, 22]. Previously, the $\phi$ production in nuclei was studied by the KEK-PS-E325 Collaboration [23], the LEPS Collaboration at Spring8/Osaka [24], the CLAS Collaboration at the Jefferson Lab [25], and very recently by the ANKE experiment at COSY-Jülich [27]. Together with theoretical predictions [26, 27] on the nuclear transparency ratio it has been concluded that the $\phi$ experiences a huge absorption in nuclei, consistent with inelastic widths about one order of magnitude higher than its dominant decay in vacuum. These results point at medium effects typically larger than predicted by hadronic models, leaving room for additional interaction processes with the nuclear medium. A possible mechanism increasing the $\phi$ absorption would rely on the $\phi N$ interaction mediated by vector meson exchange. Such interaction was explored recently in vacuum within the chiral unitary approach of [15] in the $S=0$ sector. The generation of a $N^{*}$ resonance around $2 \mathrm{GeV}$ in this approach (right above the $\phi N$ threshold) may lead to a large selfenergy contribution, further modifying the $\phi$ spectral function. 

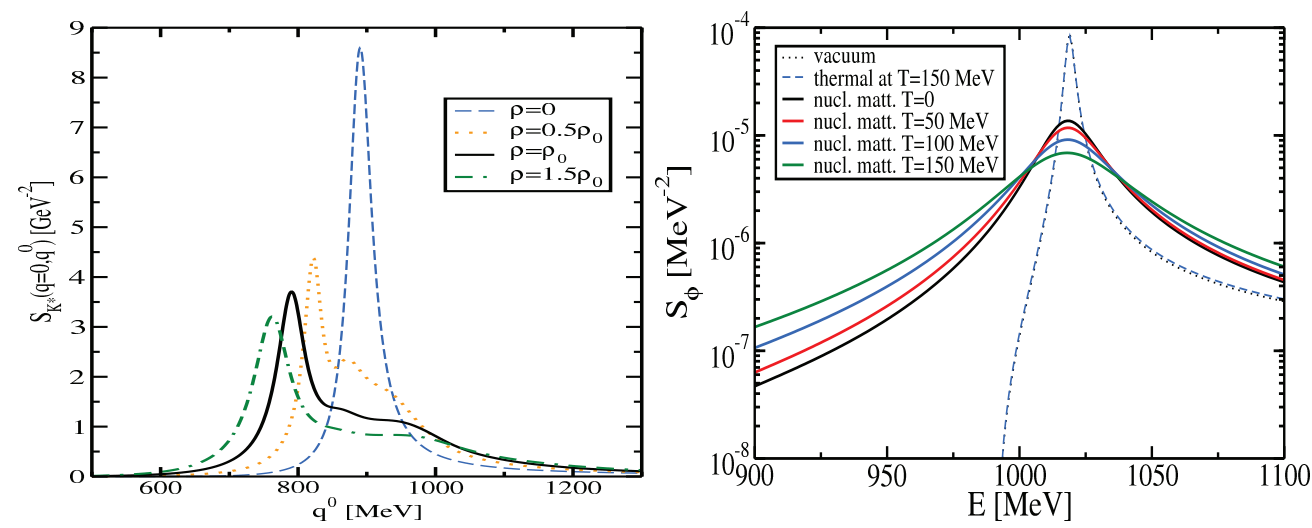

Figure 4. Left: Spectral function of the $\bar{K}^{*}$ including both collisional and $\bar{K} \pi$ selfenergies in dense nuclear matter at several densities. Right: $\phi$ meson spectral function at nuclear matter density and several temperatures, including medium effects from $\bar{K} K$ decays.

Application of the former theoretical studies in a scenario of hot and dense nuclear matter within transport approaches requires additional developments, particularly the extension of hadronic models to finite temperature. We briefly discuss some results on the two-meson cloud contribution to the in-medium width of strange vector mesons including the $\phi$. The in-medium width (accounting for inelastic or absorption channels) can be obtained straightforwardly from the imaginary part of the vector-meson selfenergy $\left(\Gamma_{\bar{K}^{*} \rightarrow \bar{K} \pi}=-\operatorname{Im} \Pi_{\bar{K}^{*} \bar{K} \pi} / P^{0}\right)$, which for a $\bar{K}^{*}$ at rest reads

$$
\operatorname{Im} \Pi_{\bar{K}^{*} \bar{K} \pi}\left(P^{0}, \overrightarrow{0}\right)=-2 \pi g^{2} \int \frac{\mathrm{d}^{3} q \vec{q}^{2}}{(2 \pi)^{3}} \int_{0}^{P^{0}} \mathrm{~d} \omega S_{\bar{K}}(\omega, \vec{q}) S_{\pi}\left(P^{0}-\omega, \vec{q}\right)\left[1+f_{\omega}+f_{P^{0}-\omega}\right]+\text { diff. }
$$

The coupling $g$ is fixed to reproduce the $\bar{K}^{*}$ vacuum decay width and $f_{\omega}$ stands for the Bose distribution function (similar expressions are obtained for $K^{*}$ and $\phi$ mesons). $S_{\bar{K} / \pi}$ denotes the pseudoscalar meson spectral function, which is given by the imaginary part of the meson propagator and depends explicitly on the meson selfenergy, namely $S\left(q^{0}, \vec{q}\right)=-\pi^{-1} \operatorname{Im}\left[\left(q^{0}\right)^{2}-\vec{q}^{2}-m^{2}-\Pi\left(q_{0}, \vec{q}\right)\right]^{-1}$. The omitted terms in Eq. (2), labelled as diff, stand for diffusive contributions in which the $\bar{K}^{*}$ can be absorbed by a thermally excited meson, namely $\bar{K}^{*} \pi \rightarrow \bar{K}$ and $\bar{K}^{*} K \rightarrow \pi$. They are small at the nominal $\bar{K}^{*}$ mass but may contribute notably at low (off-shell) energies. The Bose enhancement factor becomes important when the light pseudoscalars develop low-energy modes as a consequence of their in-medium dressing (as is the case for pions and $\bar{K}$ 's in baryon-rich matter).

The evolution of the $\bar{K}^{*}$ and $K^{*}$ in-medium decay width with the energy, temperature and density is depicted in Fig. 3. The results exhibit a dramatic enhancement over the vacuum case $\left(\Gamma_{K^{*}}^{\mathrm{vac}}=42 \mathrm{MeV}\right)$ even for the $K^{*}$, mostly originating in the broadening of the pion and the population of low-energy modes. It is worth mentioning that the HADES collaboration have recently reported an important reduction of the $K^{* 0}$ yield in $\mathrm{Ar}+\mathrm{KCl}$ collisions at $1.76 \mathrm{~A} \mathrm{GeV}$ when compared to estimations within the UrQMD transport approach $[8,28]$. In the ultrarelativistic regime the ALICE Collaboration have recently reported the suppression of $K^{*} / \bar{K}^{*}$ production in $\mathrm{Pb}+\mathrm{Pb}$ collisions at $\sqrt{s_{N N}}=2.76 \mathrm{TeV}$. The STAR Collaboration measurements of $K^{*} / \bar{K}^{*}$ hadronic decays in $\mathrm{Cu}+\mathrm{Cu}$ and $\mathrm{Au}+\mathrm{Au}$ collisions at two different energies suggest a major role of rescattering by the decay daughters of the vector mesons in the late hadronic medium. 
In Fig. 4(left) the spectral function of the $\bar{K}^{*}$ in nuclear matter at several densities is shown as reported in [17]. Several resonance-hole excitations are visible together with the quasi-particle peak, which is dramatically shifted to lower energies, revealing a complicated many-body structure of the vector-meson selfenergy. On the right panel of Fig. 4 we show the $\phi$ spectral function in hot and dense nuclear matter with $K$ and $\bar{K}$ dressing as reported in [11] (the small mass shift obtained for the $\phi$ in [29] is neglected here). The $\phi$ is considerably broadened at the quasiparticle peak with a sizable dependence on the temperature of the medium. An enhancement of the spectral function is visible at low energies below the nominal $\bar{K} K$ threshold. The population of this region originates in the opening of decay channels such as $\phi N \rightarrow \Lambda, \Sigma, \Sigma^{*}$. Further conclusions on present and future measurements of strange vector mesons from HICs can be drawn from detailed analysis within transport approaches incorporating realistic spectral functions with medium effects as discussed here. Such developments are in progress.

\section{Acknowledgements}

This work has been supported by the Helmholtz International Center for FAIR within the framework of the LOEWE program. We also acknowledge support from Grants No. FPA2010-16963 (Ministerio de Ciencia e Innovación), No. FP7-PEOPLE-2011-CIG under Contract No. PCIG09-GA-2011-291679, No. FPA2013-43425-P, and the European Community-Research Infrastructure Integrating Activity Study of Strongly Interacting Matter (acronym HadronPhysics3, Grant Agreement n. 283286) under the Seventh Framework Programme of EU. D.C. acknowledges support from the BMBF (Germany) under project No. 05P12RFFCQ. L.T. acknowledges support from the Ramón y Cajal Research Programme (Ministerio de Ciencia e Innovación).

\section{References}

[1] E. Friedman and A. Gal, Phys. Rept. 452, 89 (2007).

[2] C. Fuchs, Prog. Part. Nucl. Phys. 56, 1 (2006).

[3] A. Forster et al., Phys. Rev. C 75, 024906 (2007).

[4] C. Hartnack, H. Oeschler, Y. Leifels, E. L. Bratkovskaya and J. Aichelin, Phys. Rept. 510, 119 (2012).

[5] D. B. Kaplan and A. E. Nelson, Phys. Lett. B 175, 57 (1986).

[6] W. Cassing, L. Tolos, E. L. Bratkovskaya and A. Ramos, Nucl. Phys. A 727, 59 (2003).

[7] C. Blume and C. Markert, Prog. Part. Nucl. Phys. 66 (2011) 834.

[8] G. Agakishiev et al. [HADES Collaboration], Eur. Phys. J. A 49 (2013) 34.

[9] J. Schaffner-Bielich, Phys. Rev. Lett. 84 (2000) 3261.

[10] L. Tolos, A. Ramos and E. Oset, Phys. Rev. C 74, 015203 (2006).

[11] L. Tolos, D. Cabrera and A. Ramos, Phys. Rev. C 78 (2008) 045205.

[12] D. Cabrera, L. Tolós, J. Aichelin and E. Bratkovskaya, Phys. Rev. C 90 (2014) 5, 055207.

[13] M. M. Kaskulov and E. Oset, Phys. Rev. C 73, 045213 (2006).

[14] E. Oset and A. Ramos, Eur. Phys. J. A 44 (2010) 445.

[15] E. Oset, A. Ramos, E. J. Garzon, R. Molina, L. Tolos, C. W. Xiao, J. J. Wu and B. S. Zou, Int. J. Mod. Phys. E 21 (2012) 1230011.

[16] A. Ramos and E. Oset, Phys. Lett. B 727 (2013) 287.

[17] L. Tolos, R. Molina, E. Oset and A. Ramos, Phys. Rev. C 82 (2010) 045210.

[18] A. Ilner, D. Cabrera, P. Srisawad and E. Bratkovskaya, Nucl. Phys. A 927 (2014) 249. 
[19] A. Ilner, D. Cabrera and E. Bratkovskaya, these proceedings.

[20] K. P. Khemchandani, A. M. Torres, F. S. Navarra, M. Nielsen and L. Tolos, arXiv:1406.7203 [nucl-th].

[21] M. Wada [STAR Collaboration], Nucl. Phys. A 904-905 (2013) 1019c.

[22] B. B. Abelev et al. [ALICE Collaboration], arXiv:1404.0495 [nucl-ex].

[23] R. Muto et al. [KEK-PS-E325 Collaboration], Phys. Rev. Lett. 98 (2007) 042501.

[24] T. Ishikawa, D. S. Ahn, J. K. Ahn, H. Akimune, W. C. Chang, S. Date, H. Fujimura and M. Fujiwara et al., Phys. Lett. B 608 (2005) 215.

[25] M. H. Wood et al. [CLAS Collaboration], Phys. Rev. Lett. 105 (2010) 112301.

[26] D. Cabrera, L. Roca, E. Oset, H. Toki and M. J. Vicente Vacas, Nucl. Phys. A 733 (2004) 130.

[27] M. Hartmann, Y. T. Kiselev, A. Polyanskiy, E. Y. Paryev, M. Buscher, D. Chiladze, S. Dymov and A. Dzyuba et al., Phys. Rev. C 85 (2012) 035206.

[28] M. Bleicher, E. Zabrodin, C. Spieles, S. A. Bass, C. Ernst, S. Soff, L. Bravina and M. Belkacem et al., J. Phys. G 25 (1999) 1859.

[29] D. Cabrera and M. J. Vicente Vacas, Phys. Rev. C 67 (2003) 045203. 
